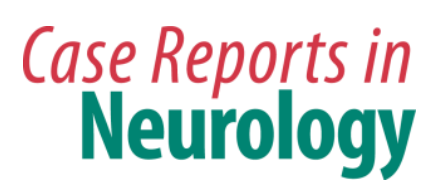

Case Rep Neurol 2013;5:91-97

\title{
Radiation-Induced Cavernoma after Total Body Irradiation and Haematopoietic Stem Cell Transplantation in an Adult Patient Suffering from Acute Myeloid Leukaemia
}

\author{
Julia Walch $^{\mathrm{a}}$ Barbara Tettenborn $^{\mathrm{a}}$ Johannes Weber ${ }^{\mathrm{b}}$ \\ Thomas Hundsberger ${ }^{a, c}$ \\ ${ }^{a}$ Department of Neurology, ${ }^{b}$ Institute of Radiology and ${ }^{c}$ Division of Haematology and \\ Oncology, Cantonal Hospital St. Gallen, St. Gallen, Switzerland
}

\section{Key Words}

Radiation-induced cavernoma $\cdot$ De novo cavernoma $\cdot$ Total body irradiation

\begin{abstract}
Cerebral cavernomas are thin-walled vascular lesions composed of dilated capillary spaces. De novo formation of cavernomas after cerebral radiotherapy has been suspected since 1994. They are mostly seen in children after irradiation of brain tumours. Radiation dose and the developing juvenile brain are predisposing factors causing cavernomas. However, the underlying mechanisms are still far from being understood. In adults, radiation-induced cavernomas (RICs) usually occur 10 years after a high cumulative radiation dosage of $>30 \mathrm{~Gy}$. Here, we report a 45-year-old man with new-onset focal epileptic seizures caused by a haemorrhagic lesion noted on cerebral computed tomography scan. Brain MRI showed the typical appearance of a ruptured cavernoma. Of note, a cerebral MRI scan 5 years earlier showed no corresponding lesion. The patient had been treated with haematopoietic stem cell transplantation for acute myeloid leukaemia (AML) 16 years before. As part of this procedure, total body irradiation (TBI) consisting of 12 Gy was administered. According to the data from the literature, the typical delay from irradiation and a former normal brain MRI scan, we assume that our patient suffers from a RIC. To our knowledge, this is the first documented adult AML patient with a RIC treated with TBI. We aim to increase awareness
\end{abstract}


among neurologists for the association of cranial irradiation or TBI and de novo cavernomas in patients suffering from malignant diseases.

\section{Introduction}

Cavernomas are vascular lesions composed of thin-walled, dilated capillary spaces with no intervening brain tissue [1]. They are mostly of congenital origin. The prevalence of cavernomas in the general population is $0.5-0.6 \%[2,3]$. They become symptomatic either through epileptic seizures or focal neurological symptoms attributable to rupture and subsequent bleeding. The prospective haemorrhage rate has been estimated to be $0.7-4.2 \%$ [4]. Localisation of the cavernoma plays an important role in the risk of epileptic seizures. Cavernomas in the frontal lobe or in arterial border zones have a high risk [5].

De novo cavernomas are known to develop in response to cranial irradiation for the treatment of primary brain tumours, prophylactic CNS radiotherapy for acute lymphoblastic lymphoma and total body irradiation (TBI) as conditioning therapy for haematopoietic stem cell transplantation (HSCT) [6, 7]. They were first reported in 1994 [8].

The pathophysiological mechanisms of radiation-induced cavernomas (RICs) remain elusive. As children treated with cranial radiotherapy or TBI are at higher risk, hypotheses assume that the immature brain of paediatric patients is more vulnerable to actinic harm [1, 7]. Radiotherapy induces vessel wall necrosis leading to endothelial swelling, dilation of the vessel lumen, hyalinization and fibroses which predisposes to cavernoma formation [1, 7]. Others speculate that tiny, invisible cavernomas are already present before radiotherapy and growth is subsequently triggered. Finally, expression of vascular endothelial growth factor (VEGF) is increased in response to irradiation $[1,7,9]$. This potent proangiogenic factor may promote the formation of vascular abnormalities. Taken together, RICs evolve from a multitude of interplaying factors.

Heckl et al. [6] analysed 189 cerebral cavernomas, including a subgroup of patients treated with prior cerebral radiotherapy. Results indicated a correlation between cranial irradiation and cavernoma formation, especially in younger children at the time of radiotherapy. The majority of adult de novo cavernomas occurred after radiation doses $>30 \mathrm{~Gy}$ [6]. The median age at the time of cavernoma detection varies between 8 and 11.7 years in unselected patient cohorts [2, 7]. In children, incidence and development of de novo cavernomas are associated with radiation dosage [10]. The interval between cranial radiotherapy and the detection of de novo cavernomas is approximately 8 years $[2,7]$.

\section{Case Report}

A 45-year-old man was admitted to our hospital with a series of focal and generalized epileptic seizures. Initial clinical presentation showed a disoriented patient with left-sided tongue bite. Neurological examination disclosed paresis of the left hand. Laboratory results were normal apart from low potassium and low haemoglobin levels. ECG and thoracic X-ray were also unremarkable. Computed tomography of the brain showed a haemorrhagic partially calcified lesion in the right parietal lobe. Subsequent MRI studies revealed a haemorrhagic transformed and space-occupying lesion diagnosed as a cavernoma (fig. 1, fig. 2). According to the clinical symptoms and the anatomical correlation, the cavernoma was the underlying lesion responsible for the new onset of focal epileptic seizures. 
The patient's previous medical history included intensive treatment with various chemotherapeutic agents including cytarabine/idarubicin (first cycle), cytarabine/amsacrine (second cycle) and cytarabine/etoposide (third cycle) for the treatment of acute myeloid leukaemia (AML), subtype M2, in 1999. After early bone marrow recurrence in March 2000, he received reinduction chemotherapy (S-HAM) and an allogenic HSCT in July 2000. As part of the therapy, he was treated with TBI with 12 Gy in 6 fractions. After diverse infections (sinusitis, invasive pulmonary aspergillosis and mucositis) he finally achieved complete remission. Later on, he suffered from treatment-related secondary arterial hypertension most probably due to cyclosporine-associated renal impairment. He frequently complained about headaches which led to a first cerebral MRI in 2006 to exclude secondary causes. The MRI scan was entirely normal, without evidence of vascular lesions (fig. 3). Until 2011, neither a neurological evaluation nor a further radiological evaluation was performed.

According to the well-documented history including former cerebral MRI studies, we speculate that the newly diagnosed cavernoma in 2011 was triggered by TBI.

\section{Discussion}

Here, we report a 45-year-old patient with a RIC after intensive and multimodal treatment for AML. The development of a cerebral RIC is underlined by an entirely normal highresolution cerebral MRI 5 years before the diagnosis. Several aspects of the patient are noteworthy.

Patients irradiated for the treatment of medulloblastomas, gliomas and acute lymphoblastic lymphoma are predominantly at risk for the development of RICs [6, 7]. However, a growing body of evidence indicates that not only primary brain tumours but also haematological diseases treated with any cerebral radiotherapy are prone to the risk of cerebral cavernoma development. Faraci et al. [10] investigated a population of children $(n=56)$ between 3 and 14 years of age who had received radiotherapy for leukaemia. Patients either received 18-24 Gy for cranial irradiation or 9.9-12 Gy for TBI before HSCT. Cerebral cavernoma was the most frequent finding in $57 \%$ of the patients 11 years after therapy. The cohort contained 4 children with AML.

Another study investigated 68 patients with various haematological malignancies treated with radiotherapy in the context of HSCT. The median age for the entire study cohort was 7 years (range 0-24) and the median age at follow-up was 28 years (range 14-43). The cohort contained 11 cases of AML. All of these AML patients were subdivided in a heterogeneous patient group irradiated with 6-12 Gy as a conditioning therapy for HSCT ( $\mathrm{n}=39$ ). The median age for this group was 9 years (range 0-24). Development of a RIC was detected in $46.2 \%$ of these patients. A detailed analysis of the AML patients was not provided.

Therefore, we present here the first adult AML patient undergoing HSCT in combination with TBI with a documented history of a RIC. Furthermore, our patient is much older compared to most other reports, as RICs mainly develop in the second decade [7, 11]. TBI with a cumulative radiation dose of 12 Gy as a trigger for a RIC is considerably low. An analysis of 15 patients older than 10 years of age indicated that the vast majority of the patients received radiation doses $\geq 30$ Gy [6]. However, in the same report, the authors demonstrated a possible RIC in a region with radiation doses $<18 \mathrm{~Gy}$.

Koike et al. [3] investigated a cohort of juvenile and adolescent long-term survivors of HSCT $(n=19)$ who did or did not receive radiotherapy as part of conditioning treatment. They demonstrated that $46.2 \%(n=36)$ of the patients irradiated with 6-12 Gy developed 
cerebral RICs. All patients treated with 18-36 Gy developed cavernomas. Nonirradiated patients did not develop any cerebral cavernomas. This clearly discloses cerebral irradiation itself, and higher doses in particular, as a risk factor in the context of HSCT [2, 3]. The probability of developing a RIC 25 years after HSCT was $61.5 \%$ in 28 out of 68 children. Radiation dosages of 6-12 Gy might be considered as the threshold level for cavernoma formation. Moreover, Faraci et al. [10] reported that a radiation dose $<1$ Gy might be sufficient to trigger VEGF release and induce neoangiogenesis leading to cavernomas. These data fit well with the low radiation dosage applied to our patient reported herein.

Controversially, a retrospective analysis of 552 patients ( $<18$ years) did not confirm a correlation between radiation dose and cerebral cavernoma formation within the radiation field [12]. These authors identified various neoplastic lesions within a certain distance of the maximal irradiation area. It was speculated that cells with proliferative potential are killed in the high radiation areas compared to sublethal DNA damage of cells in low-dose areas. Sublethal actinic harm may trigger abnormal cell growth and the formation of RICs.

The median time from irradiation to cerebral cavernoma formation is reported to be 8.9 years [7]. This is 7 years earlier than in the case described herein. One could speculate that the lower radiation dose may have accounted for the longer time span. This is supported by the fact that higher radiation doses lead to earlier formation of cerebral cavernomas in children $<10$ years of age [6].

The prevalence of RICs is not known. However, possible risk factors are discussed in the literature. An inherited susceptibility of the immature brain of children might be an issue. An analysis of 40 patients with RICs revealed that half are detected in the age group between 10 and 19 years [6]. Additionally, a literature review of 84 cases with RICs reported a median age of 8 years at the time of radiation and a median interval to cavernoma formation of 8 years [2]. Younger age at irradiation resulted in a shorter period of time to cavernoma development. Koike et al. [3] investigated several risk factors in patients undergoing HSCT. Gender, type of transplantation and age at irradiation were not significant risk factors. Interestingly, patients over 15 years of age at irradiation had a marginally higher risk than younger patients. However, this correlation was explained with higher radiation doses applied in older children.

CNS irradiation leads to several vascular effects such as endothelial swelling and adventitial vessel proliferation, causing focal ischemia. Hypoxia stimulates expression of hypoxiainducible factor-1 and VEGF; the latter being the strongest proangiogenic factor [6]. Higher expression of these biological factors has been demonstrated in younger patients, which partially explains susceptibility of vascular malformations in response to radiotherapy in children. Taken together, higher radiation dose and younger patient age are negative risk factors for the development of RICs.

Since our patient received TBI, one would probably expect multiple cavernomas in the body or at least in the brain. However, most patients develop only a single cavernoma, whereas multiple cavernomas are extraordinary rare [12]. This observation supports the hypothesis of a preexisting vascular lesion not detectable on MRI scans as the predisposing factor for a radiotherapy-induced proliferation trigger for cavernomas.

An important issue in intracerebral cavernomas is the risk for acute or subacute haemorrhages. Flemming et al. [4] demonstrated in a cohort of incidental cavernomas that the annual rate of bleeding was $6.19 \%$ in patients initially presenting with haemorrhage, $2.18 \%$ in patients with symptoms not related to haemorrhage and $0.33 \%$ in patients with an incidental finding. Prior haemorrhage, multiplicity of cavernomas and pregnancy were negative risk factors for bleeding [3]. Our patient had none of these risk factors apart from arterial hypertension. It was also shown that infratentorial cavernomas are more likely to be 
associated with symptomatic bleeding compared to supratentorial cavernomas [3]. This can easily be explained due to the anatomical characteristics of the posterior fossa. Of note, de novo cavernomas have an increased risk for bleeding compared to congenital cavernomas but are mainly located supratentorially [2].

In conclusion, cerebral cavernomas should be considered in the differential diagnosis of focal neurological symptoms in any patient who has received previous cranial radiotherapy. This is especially true for patients treated with HSCT and TBI for haematological disorders. The case reported here accumulates evidence that a cumulative radiation dosage $>30$ Gy has to be taken into account as a risk factor for cerebral cavernoma formation also in adults $[3$, 6].

\section{Disclosure Statement}

The authors declare no conflict of interest.

\section{References}

$\rightarrow 1$ Jain R, Robertson PL, Gandhi D, Gujar SK, Muraszko KM, Gebarski S: Radiation-induced cavernomas of the brain. AJNR Am J Neuroradiol 2005;26:1158-1162.

-2 Keezer MR, Del Maestro R: Radiation-induced cavernous hemangiomas: case report and literature review. Can J Neurol Science 2009;36:303-310.

-3 Koike T, Yanagimachi N, Ishiguro H, Yabe H, Yabe M, Morimoto T, Shimizu T, Takakura H, Kato S: High incidence of radiation-induced cavernous hemangioma in long-term survivors who underwent hematopoietic stem cell transplantation with radiation therapy during childhood or adolescence. Biol Blood Marrow Transplant 2012;18:1090-1098.

4 Flemming KD, Link MJ, Christianson TJ, Brown RD Jr: Prospective hemorrhage risk of intracerebral cavernous malformations. Neurology 2012;78:632-636.

5 Garcin B, Houdart E, Porcher R, Manchon E, Saint-Maurice JP, Bresson D, Stapf C: Epileptic seizures at initial presentation in patients with brain arteriovenous malformation. Neurology 2012;28:626-631.

6 Heckl S, Aschoff A, Kunze S: Radiation-induced cavernous hemangiomas of the brain: a late effect predominantly in children. Cancer 2002;94:3285-3291.

-7 Nimjee SM, Powers CJ, Bulsara KR: Review of the literature on de novo formation of cavernous malformations of the central nervous system after radiation therapy. Neurosurg Focus 2006;21:e4.

-8 Ciricillo SF, Cogen PH, Edwards MS: Pediatric cryptic vascular malformations: presentation, diagnosis and treatment. Pediatr Neurosurg 1994;20:137-147.

-9 Strenger V, Sovinz P, Lackner H, Dornbusch HJ, Lingitz H, Eder HG, Moser A, Urban C: Intracerebral cavernous hemangioma after cranial irradiation in childhood. Incidence and risk factors. Strahlenther Onkol 2008;184:276-280.

10 Faraci M, Morana G, Bagnasco F, Barra S, Polo P, Hanau G, Fioredda F, Caruso S, Rossi A, Spaziante R, Haupt R: Magnetic resonance imaging in childhood leukemia survivors treated with cranial radiotherapy: a cross sectional, single center study. Pediatr Blood Cancer 2011;57:240-246.

11 Motegi H, Kuroda S, Ishii N, Aoyama H, Terae S, Shirato H, Iwasaki Y: De novo formation of cavernoma after radiosurgery for adult cerebral arteriovenous malformation. Neurol Med Chir (Tokyo) 2008;48:397-400.

12 Vinchon M, Leblond P, Caron S, Delestret I, Baroncini M, Coche B: Radiation-induced tumors in children irradiated for brain tumor: a longitudinal study. Childs Nerv Syst 2011;27:445-453. 


\section{Case Reports in Neurology}

\begin{tabular}{l|l}
\hline \multicolumn{2}{l}{ Case Rep Neurol 2013;5:91-97 } \\
\hline DOI: $10.1159 / 000351069$ & $\begin{array}{l}\text { C 2013 S. Karger AG, Basel } \\
\text { www.karger.com/crn }\end{array}$ \\
\hline
\end{tabular}

Walch et al.: Radiation-Induced Cavernoma after Total Body Irradiation and

Haematopoietic Stem Cell Transplantation in an Adult Patient Suffering from Acute Myeloid Leukaemia

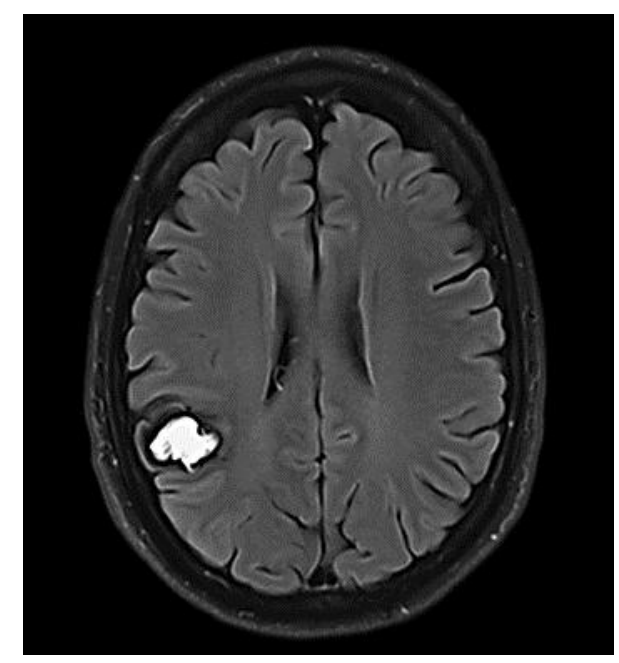

Fig. 1. Five years after the first inconspicuous MRI scan and 11 years after radiation therapy, a typical cavernoma with surrounding haemosiderin- and methaemoglobin-containing caverns, reflecting former haemorrhage, was detected in the FLAIR MRI sequence.

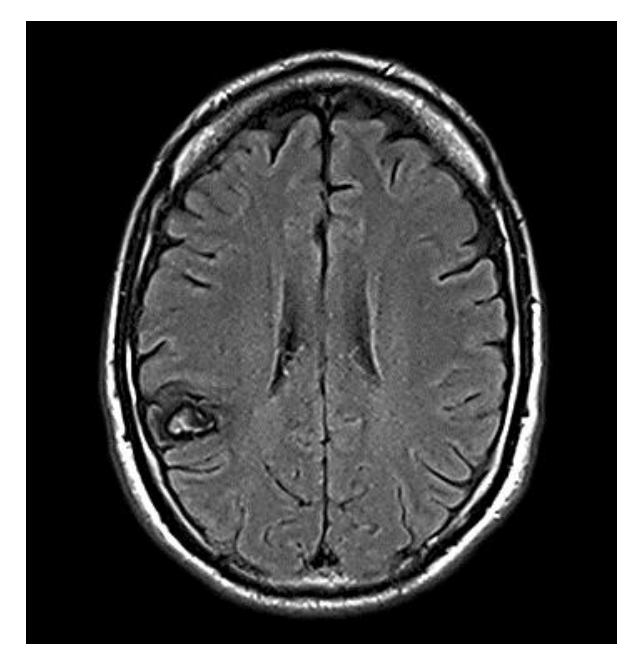

Fig. 2. The follow-up MRI (FLAIR) performed 4 months after the 5-year follow-up MRI revealed significant decrease in size with residual haemorrhagic changes of the cavernoma. 
Walch et al.: Radiation-Induced Cavernoma after Total Body Irradiation and

Haematopoietic Stem Cell Transplantation in an Adult Patient Suffering from Acute Myeloid Leukaemia

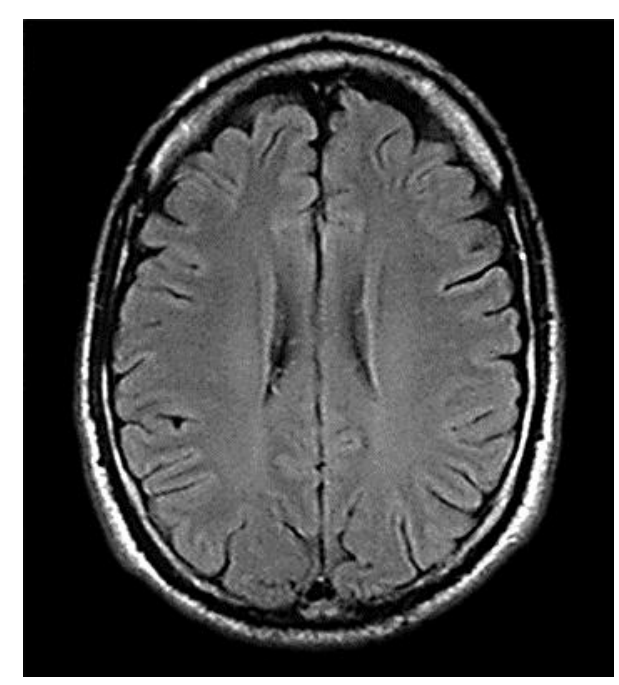

Fig. 3. In a cranial MRI performed in 2006, the FLAIR sequence revealed no pathological lesions anywhere in the brain. In particular, the right-sided supramarginal gyrus, where the cavernoma would later develop, shows no evidence of any signal alteration. 